\title{
Throughput and Stability of Digital and Analog Network Coding for Wireless Networks with Single and Multiple Relays
}

\author{
(Invited Paper)
}

\author{
Yalin Evren Sagduyu \\ Electrical Engineering and \\ Computer Science \\ Northwestern University \\ Evanston, IL 60208, USA \\ y-sagduyu@ \\ northwestern.edu
}

\author{
Dongning Guo \\ Electrical Engineering and \\ Computer Science \\ Northwestern University \\ Evanston, IL 60208, USA \\ dguo@northwestern.edu
}

\author{
Randall Berry \\ Electrical Engineering and \\ Computer Science \\ Northwestern University \\ Evanston, IL 60208, USA \\ rberry@ece. \\ northwestern.edu
}

\begin{abstract}
We evaluate the throughput and stability properties of digital and analog network coding for wireless terminals exchanging broadcast traffic with the assistance of relay nodes. For stochastically varying traffic, the stable operation is compared under the different schemes of plain routing, and digital and analog network coding, at the packet and the signal levels, respectively. For each network coding scheme, the queue dynamics are specified for the terminal and relay nodes, and the maximum throughput region is optimized over all transmission schedules. We then give generalizations of the well-known back-pressure policy for each scheme, which accounts for the coupling of the queues due to network coding and is throughput optimal, i.e., it stabilizes the network whenever this is possible. Our initial analysis focuses on a network with a single relay node. We then discuss extensions of this to arbitrary terminal-relay configurations in a general multihop network. A general framework is established to construct the maximum throughput region and throughput optimal scheduling is jointly designed with network coding for relay networks with general channel rates. For any achievable rates in the maximum throughput region the dynamic scheduling and coding ensure that the average queue lengths at the terminal and relay nodes are asymptotically bounded.
\end{abstract}

\section{Keywords}

Analog network coding, digital network coding, queue stability, stable throughput region, throughput optimal control.

\section{INTRODUCTION}

Throughput optimal control of store-and-forward-based

Permission to make digital or hard copies of all or part of this work for personal or classroom use is granted without fee provided that copies are not made or distributed for profit or commercial advantage and that copies bear this notice and the full citation on the first page. To copy otherwise, to republish, to post on servers or to redistribute to lists, requires prior specific permission and/or a fee.

WICON '08, November 17-19, 2008, Maui, Hawaii, USA

Copyright 2008 ACM ICST 978-963-9799-36-3 ...\$5.00. plain routing for stochastically varying packet flows is realized by maximum differential backlog policies (also known as back-pressure algorithms) [1]. This approach applies to unicast communication between multiple source-destination pairs, and has also been extended to multicast communication under a joint scheduling and routing framework [2].

In this paper, we consider generalizations of such policies for broadcast traffic in networks that employ network coding. As an extension of plain routing, network coding can be viewed as cooperative communication at the network layer and allows intermediate nodes to code over the incoming packets for backlogged packet traffic [3]. Network coding can be also performed in analog form at the physical layer as an extension of the amplify-and-forward mechanism [4].

For stochastically varying packet traffic, random network coding has been adapted to back-pressure algorithms under the assumption that network coding is limited to the packets of the same source [5]. The stable operation of inter-session network coding with multiple sources has been analyzed for fixed scheduling solutions [6]. Dynamic scheduling has been specified in [7] for the special case of pairwise network coding of two unicast flows.

The queue stability with multi-source network coding has been studied for different canonical network models. For exchange channels between two sources over a single relay, the stability region has been computed in [8] for a bidirectional relaying protocol and the effects of opportunistic scheduling have been evaluated in [9] for Poisson packet arrivals. As an extension to two relay nodes, stability properties have been evaluated in [10] in conjunction with cooperative communication and network coding. Throughput optimal control has been also considered in [11] to study the queue stability of inter-session network coding for butterfly network model.

In this work, we consider local network coding at relay nodes that are relied upon to exchange broadcast traffic of multiple source terminals with arbitrary packet arrival rates. For constant backlogged traffic (where nodes always have packets available to transmit), the problem of network coding over a single relay node has been addressed in [12] in terms of achievable throughput rates without concern of stability and delay build-up in packet queues. In [13], this saturated queue model has been extended to the stable operation for stochastically varying traffic (with possibly empty packet 
queues) over error-free channels with uniform channel rates. In this paper, we generalize first the channel properties for the case of a single relay with multiple source terminals and then we consider simultaneous operation of multiple relays with arbitrary terminal assignments.

For each digital and analog network coding scheme, our objectives are: (a) Construct the maximum throughput region by optimizing the flow conditions over transmission schedules, and (b) Derive the throughput optimal control solutions to stabilize the packet queues.

Meeting these objectives is not straightforward extension of plain routing case in [1], because network coding serves packet queues jointly. Hence, the service rates of relay and terminal queues depend on each other's queue backlogs for digital and analog network coding, respectively. Lyapunov stability over instantaneous queue contents cannot be directly applied to establish the stability properties for network coding (compared to back-pressure algorithms for plain routing [1]). Instead, we consider Lyapunov stability arguments over the entire duration of any network coding session in order to decouple the packet queues. Throughput optimal control results in different formulations of back-pressure algorithms with different metrics based on the differential queue backlogs for digital and analog network coding.

We show that the packet queues can be stabilized while achieving all rates in the maximum throughput region. The approach is based on keeping virtual queues at the terminal and relay nodes, and asymptotically bounding the average queue lengths. This results in cross-layer solutions of queuebased network coding and dynamic scheduling without any a priori information on packet arrival statistics. For that purpose, the network coding solutions of [12] for the backlogged queue model need to be extended to accommodate stochastic traffic. Network coding is designed in adaptation to queue contents and requires a joint formulation with dynamic scheduling.

We quantify the improvement of stable throughput rates over plain routing for each network coding scheme. The throughput region is specified depending on the physical channel properties and the number of neighbors each terminal can overhear in the multihop operation. As the number of terminals increases, the throughput rates of different schemes approach each other for finite overhearing range.

The paper is organized as follows. The system model is introduced in Section 2 for the single relay case. We derive the maximum throughput region and find the throughput optimal control for digital and analog network coding in Sections 3 and 4, respectively. Then, we consider the general multihop operation with multiple relay nodes in Section 5 . Finally, we discuss the stable throughput gains of network coding in Section 6 and draw conclusions in Section 7.

\section{SINGLE RELAY MODEL}

We consider $K \geq 2$ terminals communicating with each other with the aid of a single relay node $R$, as shown in Figure 1. We assume broadcast communication such that the packets of each terminal have to be delivered to all other terminals. There are only two possible paths for packets either by direct transmission between terminals or by twohop transmissions through relay $R$. In particular, we do not allow terminals to forward packets received from the other terminals. We consider omnidirectional transmissions such that any relay transmission reaches all terminals and any terminal transmission reaches relay $R$. Any terminal $j$ can overhear $n$ neighbors on each side, collectively denoted as set $\mathcal{N}_{j}$. Two network coding schemes are considered:

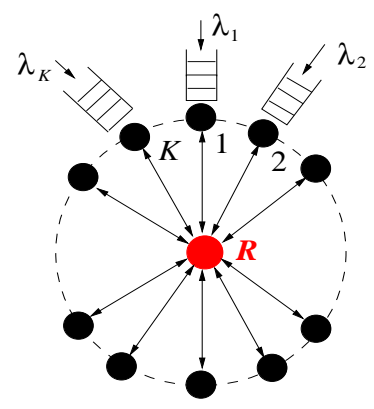

Figure 1: Star topology with $K \geq 2$ terminals and a single relay $R$.

(a) Digital Network Coding: Terminals take turn to transmit their packets to relay $R$. Relay $R$ decodes, re-encodes and broadcasts the incoming packets, assuming linear network coding in finite field $F_{q}$ with field size $q$.

(b) Analog Network Coding: Relay $R$ receives the superposition of signals from the terminals, and then amplifies and forwards it back to all terminals.

We consider the common framework of plain routing and (digital and analog) network coding, as studied in [12] for saturated packet queues. Half-duplex communication (such that relay node cannot simultaneously transmit and receive packets) is assumed in a synchronous slotted system. Therefore, we consider a two-phase operation separated in time. Let $\mathbf{x}$ define the set of terminal packets.

In the first phase, terminals transmit packets

$$
\mathbf{x}_{T}=\tilde{G} \mathbf{x},
$$

where $\tilde{G}$ is the scheduling matrix such that $\tilde{G}_{t, j}=1$, if terminal $j$ transmits in time slot $t$ of the first phase, and $\tilde{G}_{t, j}=0$, otherwise.

In the second phase, relay $R$ transmits packets

$$
\mathbf{x}_{R}=G \mathbf{y}_{R},
$$

where $\mathbf{y}_{\mathbf{R}}$ is the set of packets received by relay $R$ and $G$ is the coding matrix such that $G_{i, j}$ is the linear coding coefficient for the packet of terminal $j$ in the $i$ th coded packet. We have $\tilde{G}=I_{K}$ for plain routing and digital network coding, and $G=I_{K}$ for plain routing and analog network coding, where $I_{K}$ is the $K \times K$ identity matrix.

For plain routing and digital network coding, relay $R$ needs to decode all packets before broadcasting them (in plain or coded form) back to the terminals. We assume error-free channels and do not allow multiple packet reception at relay $R$ so that the received signal at $R$ is given by $\mathbf{y}_{R}=\mathbf{x}_{T}$. The channel rates are assumed to be constant. ${ }^{1}$ We denote by $C_{i, R}$ the capacity of the channel from terminal $i$ to relay $R$ and denote by $C_{R, i}$ the capacity of the channel from relay $R$ to terminal $i$. For packet overhearing in direct transmissions, we assume channel rate of $C_{i, j}=1$ from terminal $i$ to terminal $j$, if $i \in \mathcal{N}_{j}$, i.e., one packet can be successfully received per time slot by the overhearing terminals. Otherwise, we have $C_{i, j}=0$. On the other hand, the

\footnotetext{
${ }^{1}$ The analysis also extends to time-varying dynamic channel conditions. In this paper, we skip this extension for brevity.
} 
terminal and relay transmissions are fully coupled in analog network coding. The end-to-end channel rates over two hops will be considered in Section 4.

If $2 n \geq K-1$, any terminal transmission can reach all other terminals without need for the relay node. The optimal policy is that the terminal with the longest (non-empty) queue transmits at any time slot. ${ }^{2}$ Instead, we will assume $2 n<K-1$ for the rest of the paper.

Each terminal keeps a packet queue of infinite capacity that operates on a First-Come-First-Served basis. Relay $R$ maintains different queues for packets of different terminals under plain routing and digital network coding, but keeps a single queue for all packets under analog network coding.

We define $\lambda_{i}$ as the average arrival rate at terminal $i$ and $A_{i}(t)$ as the number of packets arriving at terminal $i$ at time slot $t$. Transmissions of terminal $i$ and relay $R$ are allocated over separate time fractions $f_{i}$ and $f_{R}$, respectively. The maximum throughput region $\Lambda$ is the convex hull of rates $\left\{\lambda_{i}\right\}_{i=1}^{K}$ that are achieved by optimizing over $\left\{f_{i}\right\}_{i=1}^{K}$ and $f_{R}$. Throughput region optimization over fixed schedules requires a priori information on packet arrival rates. For the case of unknown packet arrival statistics, we need to consider dynamic transmission schedules based on the joint contents of queues over the entire network coding session of multiple time slots.

\section{DIGITAL NETWORK CODING}

In this section, we consider the single relay model with digital network coding. We denote by $Q_{i}$ the queue of packets at terminal $i$ and by $Q_{R}^{i}$ the queue at relay $R$ for the incoming packets of terminal $i$. At time slot $t$, the backlogs of queues $Q_{i}$ and $Q_{R}^{i}$ are $U_{i}(t)$ and $U_{R}^{i}(t)$, respectively. We define $\mu_{i}(t)$ as the transmission rate from queue $Q_{i}$ of terminal $i$ at time slot $t$ and $\mu_{R}^{i}(t)$ as the transmission rate of relay $R$ from queue $Q_{R}^{i}$ for packets of terminal $i$ at time slot $t$. Note that we have $\mu_{i}(t)=0$, if $U_{i}(t)=0$, and $\mu_{R}^{i}(t)=0$, if $U_{R}^{i}(t)=0$.

Let $y_{R}^{i}$ denote the first packet in queue $Q_{R}^{i}$. The packets in relay queues are linearly coded as $G \mathbf{y}_{R}$ according to (2), if $U_{R}^{i}>0, i=1, \ldots, K$, where $\mathbf{y}_{R}=\left\{y_{R}^{i}\right\}_{i=1}^{K}$. The optimal coding matrix $G$ (with the minimum number of rows) is not unique and has been specified in [12] for backlogged traffic. A linearly independent set of $m=K-1-2 n$ coded transmissions is necessary and sufficient to exchange one packet per terminal (whereas plain routing requires $K$ coded transmissions), if all $K$ terminals are backlogged, i.e., the optimal coding matrix $G$ has the dimension of $m \times K$.

In stable operation, the service rates of relay queues are coupled in digital network coding and depend on the number of backlogged relay queues. Assume that $q_{R}$ relay queues are backlogged with at least one packet to code and transmit. If $q_{R}>m$, network coding can clear one packet from each backlogged relay queue over the next $m$ time slots, since network coding can serve up to $K$ backlogged queues over $m$ time slots, as shown in [12] (so that it would be sufficient to transmit dummy packets from the empty packet queues).

Otherwise, if $q_{R} \leq m$, plain routing is optimal and can serve up to $m$ packets from the relay queues over $m$ time slots. The backlogged terminals would be limited to plain routing, since they cannot find more than $m$ backlogged ter-

\footnotetext{
${ }^{2}$ This is similar to the longest connected queue policy for multiple terminals transmitting to a single receiver [14].
}

minals to start a network coding session.

Define $q_{R}(t)=\sum_{i=1}^{K} 1_{\left\{U_{R}^{i}(t)>0\right\}}$ as the number of backlogged relay queues at time slot $t$, where $1_{\{\cdot\}}$ is the indicator function, and define $[U]^{+}=\max (U, 0)$.

The queue lengths evolve over time as follows:

$$
\begin{gathered}
\text { (i) If } \mu_{R}^{i}(t)=0, i=1, \ldots, K, \\
U_{i}(t+1)=\left[U_{i}(t)-\mu_{i}(t)\right]^{+}+A_{i}(t), \\
U_{R}^{i}(t+1)=U_{R}^{i}(t)+\mu_{i}(t), \\
(i i) \text { If } \mu_{i}(t)=0, i=1, \ldots, K, \text { and } q_{R}(t)>m, \\
U_{i}(t+m)=U_{i}(t)+\sum_{\tau=t}^{t+m-1} A_{i}(\tau), \\
U_{R}^{i}(t+m)=\left[U_{R}^{i}(t)-\mu_{R}^{i}\left([\tau]_{\tau=t}^{t+m-1}\right)\right]^{+}, \\
(i i i) \quad \text { If } \mu_{i}(t)=0, i=1, \ldots, K, \text { and } q_{R}(t) \leq m, \\
U_{i}(t+1)=U_{i}(t)+A_{i}(t), \\
U_{R}^{i}(t+1)=\left[U_{R}^{i}(t)-\mu_{R}^{i}(\tau)\right]^{+},
\end{gathered}
$$

where $\mu_{R}^{i}\left([\tau]_{\tau=t}^{t+m-1}\right)$ is the service rate of relay queue $Q_{R}^{i}$ achievable over $m$ time slots of interval $[\tau]_{\tau=t}^{t+m-1}$ starting from $t$. Here, $(i)$ corresponds to the case where only one terminal transmits to relay $R,(i i)$ corresponds to the case where relay $R$ transmits network-coded packets to terminals over $m$ time slots, and ( $i i i$ ) corresponds to the case where relay $R$ forwards plain packets to terminals one at a time.

\subsection{Maximum Throughput Region}

THEOREM 1. For digital network coding, the maximum throughput region $\Lambda$ is given by

$$
\sum_{i=1}^{m} \frac{\lambda_{i}}{C_{R}^{i}}+\sum_{i=1}^{K} \frac{\lambda_{i}}{C_{i, R}}<1 \quad \text { for } \quad \frac{\lambda_{1}}{C_{R}^{1}} \geq \ldots \geq \frac{\lambda_{K}}{C_{R}^{K}} \geq 0,
$$

where

$$
C_{R}^{i}=\min _{j: j \neq i, i \notin \mathcal{N}_{j}} C_{R, j}, \quad i=1, \ldots, K .
$$

Proof. The rates $\left\{\lambda_{i}\right\}_{i=1}^{K}$ are in the maximum throughput region $\Lambda$, if and only if for arbitrarily small constant $\epsilon$ there exists a stationary control policy such that the flow conditions satisfy

$$
\mathbb{E}\left[\mu_{i}(t) \mid \mathbf{U}(t)\right]=\epsilon+\lambda_{i} \leq f_{i} C_{i, R}
$$

for terminal queue $Q_{i}, i=1, \ldots, K$, where $f_{i}$ is specified by the control policy, and

$$
\mathbb{E}\left[\mu_{R}^{i}\left([\tau]_{\tau=t}^{t+m-1}\right)-\sum_{\tau=t}^{t+m-1} \mu_{i}(\tau) \mid \mathbf{U}(t)\right]=m \epsilon
$$

for relay queue $Q_{R}^{i}, i=1, \ldots, K$. The expectations in (5)-(6) are taken with respect to the transmission schedules.

Under any optimal policy, the decision of performing network coding or plain routing will depend on how the number of the non-empty relay queues, $q_{R}$, compares to the threshold $m=K-1-2 n$. If $q_{R}>m$, network coding serves $q_{R}$ packets. Otherwise, if $q_{R} \leq m$, plain routing is used. Hence, during the fraction of time the relay is active, the service rates satisfy

$\sum_{i=1}^{K} \frac{1}{C_{R}^{i}} \mathbb{E}\left[\mu_{R}^{i}\left([\tau]_{\tau=t}^{t+m-1}\right) \mid \mathbf{U}(t)\right] \leq\left\{\begin{array}{l}q_{R} f_{R}, \text { if } q_{R}>m, \\ m f_{R}, \text { if } q_{R} \leq m,\end{array}\right.$ 
where $C_{R}^{i}$ in (4) is the broadcast rate for packets of terminal $i$ from relay $R$ to the rest of terminals, since each packet of terminal $i$ should be delivered to all terminals with a common rate (whereas a terminal $j$ can overhear terminal $i$ in direct transmission, if $i \in \mathcal{N}_{j}$ ). To achieve the rate (4), relay $R$ performs channel coding with respect to each terminal, and then linearly network-codes the channel-coded packets before broadcasting them back to the terminals [15].

We order relay queues such that $\frac{\lambda_{1}}{C_{R}^{1}} \geq \ldots \geq \frac{\lambda_{K}}{C_{R}^{K}}$. Relay queues $Q_{R}^{i}$ with higher values of $\frac{\lambda_{i}}{C_{R}^{i}}$ are more likely to be backlogged according to Little's law [16]. Since for $m$ relay queues to be backlogged at least one of relay queues $Q_{R}^{i}$, $i=m+1, \ldots, K$, must be backlogged, it follows that

$$
P\left(q_{R}>m\right) \geq P\left(\min _{i=m+1, \ldots, K} U_{R}^{i}>0\right)=\frac{m}{f_{R}} \sum_{i=m+1}^{K} \frac{\lambda_{i}}{C_{R}^{i}} .
$$

The service rate for the relay queue $Q_{R}^{i}, i=m+1, \ldots, K$, normalized per time slot is $\frac{f_{R}}{m} C_{R}^{i}$, since relay $R$ is activated for $f_{R}$ fraction of time and the queue is served over $m$ time slots to clear one packet. We use $P\left(q_{R}>m\right)$ to condition $q_{R} f_{R}$ in (7) for any $q_{R}=m+1, \ldots, K$. Therefore, (7) is bounded using (8) for the worst realization of $q_{R}=m+1$ (with the minimum value of $q_{R} f_{R}$ ) as follows:

$$
\begin{aligned}
& \sum_{i=1}^{K} \frac{1}{C_{R}^{i}} \mathbb{E}\left[\mu_{R}^{i}\left([\tau]_{\tau=t}^{t+m-1}\right)\right] \\
& \leq(m+1) f_{R} P\left(q_{R}>m\right)+m f_{R}\left(1-P\left(q_{R}>m\right)\right) \\
& \leq m\left(f_{R}+\sum_{i=m+1}^{K} \frac{\lambda_{i}}{C_{R}^{i}}\right) .
\end{aligned}
$$

From (5), (6), and (9), the constraints on the maximum throughput region $\Lambda=\left\{\lambda_{i}\right\}_{i=1}^{K}$ are given by

$$
\begin{aligned}
& 0 \leq \lambda_{i}<f_{i} C_{i, R}, \quad i=1, \ldots, K, \\
& \sum_{i=1}^{m} \frac{\lambda_{i}}{C_{R}^{i}}<f_{R}, i=1, \ldots, K, \text { for } \frac{\lambda_{1}}{C_{R}^{1}} \geq \ldots \geq \frac{\lambda_{K}}{C_{R}^{K}}
\end{aligned}
$$

for arbitrarily small $\epsilon$. The maximum throughput region (3) follows from optimizing (10)-(11) over the disjoint time fractions $\left\{f_{i}\right\}_{i=1}^{K}$ and $f_{R}$ such that $\sum_{i=1}^{K} f_{i}+f_{R} \leq 1$.

For plain routing, the maximum throughput region $\Lambda$ is achieved by time sharing of terminals and relay $R$ such that

$$
\sum_{i=1}^{K} \lambda_{i}\left(\frac{1}{C_{R}^{i}}+\frac{1}{C_{i, R}}\right)<1, \quad \lambda_{i} \geq 0, \quad i=1, \ldots, K .
$$

Digital network coding expands the rate conditions for the maximum throughput region $\Lambda$ by the amount of $\sum_{i=m+1}^{K} \frac{\lambda_{i}}{C_{R}^{i}}$ over the time-sharing bounds (12) of plain routing.

\subsection{Throughput Optimal Control}

The unfinished work in terminal and relay queues $Q_{i}$ and $Q_{R}^{i}, i=1, \ldots, K$, over the $m$ time slots into the future can be bounded in terms of the current unfinished work:

$$
\begin{aligned}
& U_{i}(t+m) \leq\left[U_{i}(t)-\sum_{\tau=t}^{t+m-1} \mu_{i}(\tau)\right]^{+}+\sum_{\tau=t}^{t+m-1} A_{i}(\tau) \\
& U_{R}^{i}(t+m) \leq\left[U_{R}^{i}(t)-\mu_{R}^{i}\left([\tau]_{\tau=t}^{t+m-1}\right)\right]^{+}+\sum_{\tau=t}^{t+m-1} \mu_{i}(\tau),
\end{aligned}
$$

respectively, where $\mu_{R}^{i}\left([\tau]_{\tau=t}^{t+m-1}\right)=1$ for all $i=m+$ $1, \ldots, K$, if $q_{R}(t)>m$ such that a digital network coding session starts, or $\mu_{R}^{i}\left([\tau]_{\tau=t}^{t+m-1}\right) \leq m$ for any individual $i=1, \ldots, K$, if $q_{R}(t) \leq m$ such that relay $R$ proceeds with plain routing. (13) and (14) are upper bounds only, because new arrivals may depart before the interval of $m$ time slots is finished and $\mu_{i}(\tau)$ would contribute to (14) only if $U_{i}(\tau)>0$.

THEOREM 2. For digital network coding, throughput optimal control at time slot $t$ (when there is not any ongoing digital network coding session of $m$ time slots) is given by

(a) terminal $i$ transmits a packet from queue $Q_{i}$, if

$$
\begin{aligned}
C_{i, R}\left[u_{i}(t)\right]^{+} \geq & \max \left(\left\{C_{j, R}\left[u_{j}(t)\right]^{+}\right\}_{j=1, j \neq i}^{K},\right. \\
& \left.\left\{C_{R}^{i} U_{R}^{i}(t)\right\}_{i=1}^{K}, \frac{1}{m} \sum_{i=1}^{K} \tilde{C}_{R}^{i} U_{R}^{i}(t)\right),
\end{aligned}
$$

(b) relay $R$ transmits a plain packet from queue $Q_{R}^{i}$, if

$$
\begin{aligned}
C_{R}^{i} U_{R}^{i}(t) \geq & \max \left(\left\{C_{i, R}\left[u_{i}(t)\right]^{+}\right\}_{i=1}^{K},\right. \\
& \left.\left\{C_{R}^{j} U_{R}^{j}(t)\right\}_{j=1, j \neq i}^{K}, \frac{1}{m} \sum_{i=1}^{K} \tilde{C}_{R}^{i} U_{R}^{i}(t)\right),
\end{aligned}
$$

(c) relay $R$ transmits linearly network-coded packets from queues $Q_{R}^{i}, i=1, \ldots, K$, over $m$ time slots, if

$$
\begin{aligned}
& \frac{1}{m} \sum_{i=1}^{K} \tilde{C}_{R}^{i} U_{R}^{i}(t) \\
& \geq \max _{i=1, \ldots, K}\left(C_{i, R}\left[u_{i}(t)\right]^{+}, C_{R}^{i} U_{R}^{i}(t)\right),
\end{aligned}
$$

where $u_{i}(t)=U_{i}(t)-U_{R}(t)$.

Proof. We consider Lyapunov stability arguments to establish the stability properties of packet queues. For the set of queue backlogs $\mathbf{U}(t)$ at time slot $t$, the quadratic Lyapunov function is defined as

$$
L(\mathbf{U}(t))=\sum_{i=1}^{K}\left(\left(U_{i}(t)\right)^{2}+\left(U_{R}^{i}(t)\right)^{2}\right)
$$

Since any network coding session continues $m$ time slots, we need to define from (18) the $m$-time slot Lyapunov drift:

$$
\Delta_{m}(\mathbf{U}(t))=\mathbb{E}[L(\mathbf{U}(t+m))-L(\mathbf{U}(t)) \mid \mathbf{U}(t)] .
$$

For any non-negative real numbers $V, U, \mu$ and $A$, note that $V^{2} \leq U^{2}+\mu^{2}+A^{2}-2 U(\mu-A)$, if $V \leq[U-\mu]^{+}+A$, as given in [17]. From (13)-(14), (19) can be bounded as

$$
\begin{aligned}
& \Delta_{m}(\mathbf{U}(t)) \leq m^{2}\left(2+\sum_{i=1}^{K}\left(A_{i}^{\text {max }}\right)^{2}\right) \\
& -2 \sum_{i=1}^{K} U_{i}(t) \mathbb{E}\left[\sum_{\tau=t}^{t+m-1} \mu_{i}(\tau)-\sum_{\tau=t}^{t+m-1} A_{i}(\tau) \mid \mathbf{U}(t)\right] \\
& -2 \sum_{i=1}^{K} U_{R}^{i}(t) \mathbb{E}\left[\mu_{R}^{i}\left([\tau]_{\tau=t}^{t+m-1}\right)-\sum_{\tau=t}^{t+m-1} \mu_{i}(\tau) \mid \mathbf{U}(t)\right],
\end{aligned}
$$

since we have $\mu_{i}(t) \leq 1, \mu_{R}^{i}\left([\tau]_{\tau=t}^{t+m-1}\right) \leq m, A_{i}(t) \leq A_{i}^{\max }$, and terminal and relay queues are separately served at any time slot $t$. Conditioned on $q_{R}(t)=\sum_{i=1}^{K} 1_{\left\{U_{R}^{i}(t)>0\right\}}$, the 
differential queue backlogs satisfy

$$
\begin{aligned}
& \sum_{i=1}^{K}\left(U_{i}(t)-U_{R}^{i}(t)\right) \mathbb{E}\left[\sum_{\tau=t}^{t+m-1} \mu_{i}(\tau) \mid \mathbf{U}(t)\right] \\
& \leq m \max _{i=1, \ldots, K}\left(C_{i, R}\left[U_{i}(t)-U_{R}^{i}(t)\right]^{+}\right),
\end{aligned}
$$

for terminals, and

$$
\begin{aligned}
& \sum_{i=1}^{K} U_{R}^{i}(t) \mathbb{E}\left[\mu_{R}^{i}\left([\tau]_{\tau=t}^{t+m-1}\right) \mid q_{R}(t) \leq m\right] \\
& \leq m \max _{i=1, \ldots, K}\left(C_{R}^{i} U_{R}^{i}(t)\right),
\end{aligned}
$$

if relay $R$ performs plain routing at time slot $t$, or

$$
\begin{aligned}
& \sum_{i=1}^{K} U_{R}^{i}(t) \mathbb{E}\left[\mu_{R}^{i}\left([\tau]_{\tau=t}^{t+m-1}\right) \mid q_{R}(t)>m\right] \\
& \leq \sum_{i=1}^{K} \tilde{C}_{R}^{i} U_{R}^{i}(t)
\end{aligned}
$$

if relay $R$ starts digital network coding session at time slot $t$. From (21)-(23) the right hand side of (20) is minimized by the throughput optimal control policy (15)-(17). ${ }^{3}$ From (5), (6) and (9), the Lyapunov drift (20) satisfies

$$
\Delta_{m}(\mathbf{U}(t)) \leq B-2 \tilde{\epsilon} \sum_{i=1}^{K}\left(U_{i}(t)+U_{R}^{i}(t)\right)
$$

for constants $B=m^{2}\left(2+\sum_{i=1}^{K}\left(A_{i}^{\max }\right)^{2}\right)$ and $\tilde{\epsilon}=m \epsilon$, where $\epsilon$ satisfies the flow conditions (5), (6) and (9). Then, from (24) the average congestion is bounded as

$$
\limsup _{t \rightarrow \infty} \frac{1}{t} \sum_{\tau=0}^{t-1} \sum_{i=1}^{K}\left(\mathbb{E}\left[U_{i}(\tau)\right]+\mathbb{E}\left[U_{R}^{i}(\tau)\right]\right) \leq \frac{B}{2 \tilde{\epsilon}},
$$

and system is strongly stable [17] from congestion bound (25). The throughput optimal control policy (15)-(17) minimizes the right hand side of (20) while satisfying the flow conditions (5), (6) and (9) such that all rates in the maximum throughput region $\Lambda$ given by (3) can be stabilized.

\section{ANALOG NETWORK CODING}

In this section, we extend the analysis of the single relay model to analog network coding. Terminals are scheduled to transmit according to (1) with the scheduling matrix $\tilde{G}$, where $\tilde{G}_{t, j}=1$, if terminal $j$ transmits at time slot $t$ of the network coding session. The minimum-length schedules are not unique. The scheduling matrix $\tilde{G}$ with the minimum number of rows is specified in [12] for backlogged traffic. A period of $m=K-1-2 n$ time slots is necessary and sufficient to deliver $K$ degrees of freedom to relay $R$, i.e., the optimal scheduling matrix $\tilde{G}$ has the dimension of $m \times K$.

Combine relay queues $Q_{R}^{i}, i=1, \ldots, K$, into a single queue $Q_{R}$ on a First-Come-First-Served basis such that the simultaneous transmissions arrive at relay node as a superposed signal and they are forwarded in a subsequent time slot as a single packet. Define $U_{R}(t)$ as the backlog of queue $Q_{R}$ at

\footnotetext{
${ }^{3}$ If (17) holds with $U_{R}^{i}(t)=0$ for any $i=1, \ldots, K$, a dummy packet of all zeros is coded from queue $Q_{R}^{i}$ to start the digital network coding session.
}

time slot $t$ and define $\mu_{R}(t)$ as the transmission rate of relay $R$. Note that we have $\mu_{R}(t)=0$, if $U_{R}(t)=0$.

The channel rates in two consecutive phases (from terminals to relay $R$ and from relay $R$ to terminals) are fully coupled. Therefore, we need to express the end-to-end rate for any terminal pair. Then, this rate can be partitioned among transmissions in two phases to satisfy the Max-Flow Min-Cut condition. Define $C_{i \rightarrow j}$ as the end-to-end rate from terminal $i$ to terminal $j$ over two hops through the amplifyand-forward operation by relay $R$ for $i \notin \mathcal{N}_{j} .{ }^{4}$ Then, the broadcast rate from terminal $i$ to the rest of terminals is

$$
\tilde{C}_{i}=\min _{j: j \neq i, j \notin \mathcal{N}_{i}} C_{i \rightarrow j}, \quad i=1, \ldots, K,
$$

after taking into account the overhearing effects.

In analog network coding, the service rates of terminal queues are coupled due to scheduling of simultaneous transmissions from different packet queues. Assume that $q_{T}$ terminal queues are backlogged with at least one packet to transmit. If $q_{T}>m$, network coding can clear one packet from each backlogged terminal queue over $m$ time slots. Otherwise, if $q_{T} \leq m$, plain routing is optimal and can serve up to $m$ packets from terminal queues over $m$ time slots.

Define $q_{T}(t)=\sum_{i=1}^{K} 1_{\left\{U_{i}(t)>0\right\}}$ as the number of backlogged terminal queues at time slot $t$ and define $A_{R}(t)$ as the number of packets arriving at relay queue $Q_{R}$ at time slot $t$. The queue lengths evolve over time as follows:

$$
\begin{aligned}
& \text { (i) If } \mu_{i}(t)=0, i=1, \ldots, K \\
& U_{i}(t+1)=U_{i}(t)+A_{i}(t), \\
& U_{R}(t+1)=\left[U_{R}(t)-\mu_{R}(t)\right]^{+}, \\
& \text {(ii) If } \mu_{R}(t)=0 \text { and } q_{T}(t)>m, \\
& U_{i}(t+m)=\left[U_{i}(t)-\mu_{i}\left([\tau]_{\tau=t}^{t+m-1}\right)\right]+\sum_{\tau=t}^{t+m-1} A_{i}(\tau), \\
& U_{R}(t+m)=U_{R}(t)+\sum_{\tau=t}^{t+m-1} A_{R}(\tau), \\
& \text { (iii) If } \mu_{R}(t)=0 \text { and } q_{T}(t) \leq m, \\
& U_{i}(t+1)=\left[U_{i}(t)-\mu_{i}(t)\right]^{+}+A_{i}(t), \\
& U_{R}(t+1)=U_{R}(t)+A_{R}(t)
\end{aligned}
$$

where $\mu_{i}\left([\tau]_{\tau=t}^{t+m-1}\right)$ is the service rate of terminal queue $Q_{i}$ achievable over $m$ time slots of interval $[\tau]_{\tau=t}^{t+m-1}$. Here, $(i)$ corresponds to the case where relay $R$ amplifies and forwards the received signals to terminals, $(i i)$ corresponds to the case where terminals jointly transmit to relay $R$ over $m$ time slots in analog network coding, and (iii) corresponds to the case where only one terminal transmits to relay $R$.

\subsection{Maximum Throughput Region}

THEOREM 3. For analog network coding, the maximum throughput region $\Lambda$ is given by

$$
\sum_{i=1}^{m} \lambda_{i}<\frac{\tilde{C}}{2} \quad \text { for } \quad \lambda_{1} \geq \ldots \geq \lambda_{K} \geq 0
$$

where

$$
\tilde{C}=\min _{i=1, \ldots, K} \tilde{C}_{i} .
$$

\footnotetext{
${ }^{4}$ The rate $C_{i \rightarrow j}$ strongly depends on the physical layer properties, and it has been derived in [12] for binary symmetric channels and erasure channels under backlogged traffic.
} 
Proof. Define $f_{T}$ as the total fraction of time allocated to terminal transmissions such that $f_{i} \leq f_{T}, i=1, \ldots, K$. The rates $\left\{\lambda_{i}\right\}_{i=1}^{K}$ are in the maximum throughput region $\Lambda$, if and only if for arbitrarily small constant $\epsilon$ there exists a stationary control policy such that the flow conditions satisfy

$$
\mathbb{E}\left[\mu_{i}\left([\tau]_{\tau=t}^{t+m-1}\right) \mid \mathbf{U}(t)\right]=m\left(\epsilon+\lambda_{i}\right)
$$

for terminals $i=1, \ldots, K$. Relay $R$ does not decode packets, i.e., any transmission arrives as a new packet. Hence, the channel rate from a terminal to relay $R$ is one packet per slot. Under any optimal policy, the decision of performing network coding or plain routing will depend on the number of non-empty terminal queues, $q_{T}$. For the threshold $m=$ $K-1-2 n$, if $q_{T}>m$, the service rate is $q_{T} f_{T}$. Otherwise, if $q_{T} \leq m$, the service rate is $m f_{T}$. Hence, during the fraction of time the terminals are active, the service rates satisfy

$$
\sum_{i=1}^{K} \mathbb{E}\left[\mu_{i}\left([\tau]_{\tau=t}^{t+m-1}\right) \mid \mathbf{U}(t)\right] \leq \begin{cases}q_{T} f_{T}, & \text { if } q_{T}>m \\ m f_{T}, & \text { if } q_{T} \leq m .\end{cases}
$$

We order terminal queues such that $\lambda_{1} \geq \ldots \geq \lambda_{K}$. Terminal queues with larger arrival rates are more likely to be backlogged according to Little's law [16]. Since for $m$ terminal queues to be backlogged at least one of terminal queues $Q_{i}, i=m+1, \ldots, K$, must be backlogged, it follows that

$$
P\left(q_{T}>m\right) \geq P\left(\min _{i=m+1, \ldots, K} U_{i}>0\right)=\frac{m}{f_{T}} \sum_{i=m+1}^{K} \lambda_{i} .
$$

The service rate for the terminal queue $Q_{i}, i=m+$ $1, \ldots, K$, normalized per time slot is $\frac{f_{T}}{m}$, since terminals are activated for $f_{T}$ fraction of time and the queue is served over $m$ time slots to clear one packet. We use $P\left(q_{T}>m\right)$ to condition $q_{T} f_{T}$ in (30) for any $q_{T}=m+1, \ldots, K$. Therefore, (7) is bounded using (31) for the worst realization of $q_{T}=m+1$ (with the minimum value of $q_{T} f_{T}$ ) as follows:

$$
\begin{aligned}
& \sum_{i=1}^{K} \mathbb{E}\left[\mu_{i}\left([\tau]_{\tau=t}^{t+m-1}\right)\right] \\
& \leq(m+1) f_{T} P\left(q_{T}>m\right)+m f_{T}\left(\left(1-P\left(q_{T}>m\right)\right)\right. \\
& \leq m\left(f_{T}+\sum_{i=m+1}^{K} \lambda_{i}\right)
\end{aligned}
$$

From (29) and (32), we obtain the following rate bound for terminal transmissions:

$$
\sum_{i=1}^{m} \lambda_{i}<f_{T}
$$

Then, from (33) the arrival rate at relay queue $Q_{R}$ is the total rate of analog network-coded transmissions such that

$$
\mathbb{E}\left[A_{R}(t) \mid \mathbf{U}(t)\right]=\sum_{i=1}^{m} \lambda_{i}
$$

where $\lambda_{1} \geq \ldots \geq \lambda_{K}$. Since relay $R$ is activated to transmit for $f_{R}$ fraction of time and the packets of terminal $i$ can be decoded with common rate $\tilde{C}_{i}$ by the rest of terminals, the flow condition for relay queue $Q_{R}$ is given by

$$
\mathbb{E}\left[\mu_{R}(t) \mid \mathbf{U}(t)\right]=\epsilon+\mathbb{E}\left[A_{R}(t) \mid \mathbf{U}(t)\right] \leq f_{R} \tilde{C}_{i}
$$

for $i=1, \ldots, m$. Relay $R$ amplifies and forwards the incoming signals equivalently back to all terminals. Therefore, this broadcast phase limits the achievable rate for each terminal to $\tilde{C}=\min _{i=1, \ldots, K} \tilde{C}_{i}$ (which could be improved by more advanced analog relaying schemes with end-to-end channel coding). From (29) and (33)-(35), the constraints on the maximum throughput region $\Lambda=\left\{\lambda_{i}\right\}_{i=1}^{K}$ are given by

$$
\begin{aligned}
& \lambda_{i} \geq 0, \quad i=1, \ldots, K, \quad \sum_{i=1}^{m} \lambda_{i}<f_{T}, \\
& \sum_{i=1}^{m} \lambda_{i}<f_{R}\left(\min _{i=1, \ldots, K} \tilde{C}_{i}\right) \text { for } \lambda_{1} \geq \ldots \geq \lambda_{K}
\end{aligned}
$$

for arbitrarily small $\epsilon$, where disjoint time fractions $f_{T} \geq$ 0 and $f_{R} \geq 0$ satisfy $f_{T}+f_{R} \leq 1$. Since each terminal transmission needs to be forwarded in a subsequent time slot, we have $f_{T}=f_{R}$. The maximum throughput region (27) follows from evaluating the flows conditions (36)-(37) over the time fractions $f_{T}=f_{R}=\frac{1}{2}$.

\subsection{Throughput Optimal Control}

The unfinished work in terminal queues $Q_{i}, i=1, \ldots, K$, and in relay queue $Q_{R}$ over $m$ time slots into the future can be bounded in terms of the current unfinished work:

$U_{i}(t+m) \leq\left[U_{i}(t)-\mu_{i}\left([\tau]_{\tau=t}^{t+m-1}\right)\right]^{+}+\sum_{\tau=t}^{t+m-1} A_{i}(\tau)$,
$U_{R}(t+m) \leq\left[U_{R}(t)-\sum_{\tau=t}^{t+m-1} \mu_{R}(\tau)\right]^{+}+\sum_{\tau=t}^{t+m-1} A_{R}(\tau)$,

respectively, where $\mu_{i}\left([\tau]_{\tau=t}^{t+m-1}\right)=1$ for all $i=m+1, \ldots, K$, if $q_{T}(t)>m$ such that terminals start analog network coding session for $m$ time slots, or $\mu_{i}\left([\tau]_{\tau=t}^{t+m-1}\right) \leq m$ for any individual $i=1, \ldots, K$, if $q_{T}(t) \leq m$ such that terminals transmit packets in separate time slots for plain routing.

THEOREM 4. For analog network coding, throughput optimal control at time slot $t$ (when there is not any ongoing analog network coding session of $m$ time slots) is given by

(a) terminal $i$ transmits a packet from queue $Q_{i}$, if

$$
\left[u_{i}(t)\right]^{+} \geq \max \left(\left\{\left[u_{j}(t)\right]^{+}\right\}_{j=1, j \neq i}^{K},\left[u_{T}(t)\right]^{+}, \tilde{C} U_{R}(t)\right),
$$

(b) terminals $i=1, \ldots, K$ transmit cooperatively (i.e., they start analog network coding session) over $m$ time slots, if

$$
\left[u_{T}(t)\right]^{+} \geq \max \left(\left\{\left[u_{i}(t)\right]^{+}\right\}_{i=1}^{K}, \tilde{C} U_{R}(t)\right),
$$

(c) relay $R$ forwards a packet from queue $Q_{R}$, if

$$
\tilde{C} U_{R}(t) \geq \max \left(\left\{\left[u_{i}(t)\right]^{+}\right\}_{i=1}^{K},\left[u_{T}(t)\right]^{+}\right),
$$

where $u_{T}(t)=\frac{1}{m} \sum_{i=1}^{K} U_{i}(t)-U_{R}(t)$.

Proof. Consider the quadratic Lyapunov function given by $L(\mathbf{U}(t))=\sum_{i=1}^{K}\left(U_{i}(t)\right)^{2}+\left(U_{R}(t)\right)^{2}$. From (38)-(39), the Lyapunov drift (19) over $m$ time slots can be bounded as

$$
\begin{aligned}
& \Delta_{m}(\mathbf{U}(t)) \leq m^{2}\left(2+\sum_{i=1}^{K}\left(A_{i}^{\text {max }}\right)^{2}\right) \\
& -2 \sum_{i=1}^{K} U_{i}(t) \mathbb{E}\left[\mu_{i}\left([\tau]_{\tau=t}^{t+m-1}\right)-\sum_{\tau=t}^{t+m-1} A_{i}(\tau) \mid \mathbf{U}(t)\right] \\
& -2 U_{R}(t) \mathbb{E}\left[\sum_{\tau=t}^{t+m-1}\left(\mu_{R}(\tau)-A_{R}(\tau)\right) \mid \mathbf{U}(t)\right],
\end{aligned}
$$


since we have $\mu_{i}\left([\tau]_{\tau=t}^{t+m-1}\right) \leq m, \mu_{R}(t) \leq 1, A_{i}(t) \leq A_{i}^{\text {max }}$, $A_{R}(t) \leq 1$, and terminal queues and relay queue are separately served at any time slot. Conditioned on $q_{T}(t)=$ $\sum_{i=1}^{K} 1_{\left\{U_{i}(t)>0\right\}}$, the differential queue backlogs satisfy

$$
U_{R}(t) \mathbb{E}\left[\sum_{\tau=t}^{t+m-1} \mu_{R}(\tau) \mid \mathbf{U}(t)\right] \leq m \tilde{C} U_{R}(t),
$$

for relay $R$, and satisfy

$$
\begin{aligned}
& \sum_{i=1}^{K} U_{i}(t) \mathbb{E}\left[\mu_{i}\left([\tau]_{\tau=t}^{t+m-1}\right) \mid q_{T}(t) \leq m\right] \\
& -U_{R}(t) \sum_{\tau=t}^{t+m-1} \mathbb{E}\left[A_{R}(\tau) \mid q_{T}(t) \leq m\right] \\
& \leq m \max _{i=1, \ldots, K}\left[U_{i}(t)-U_{R}(t)\right]^{+},
\end{aligned}
$$

if terminals proceed with plain routing, or satisfy

$$
\begin{aligned}
& \sum_{i=1}^{K} U_{i}(t) \mathbb{E}\left[\mu_{i}\left([\tau]_{\tau=t}^{t+m-1}\right) \mid q_{T}(t)>m\right] \\
& -U_{R}(t) \sum_{\tau=t}^{t+m-1} \mathbb{E}\left[A_{R}(\tau) \mid q_{T}(t)>m\right] \\
& \leq\left[\sum_{i=1}^{K} U_{i}(t)-m U_{R}(t)\right]^{+},
\end{aligned}
$$

if terminals start analog network coding session at time slot $t$. From (28), $\tilde{C}$ is the maximum common rate $\tilde{C}_{i}$ achievable for all terminals $i$, since relay $R$ broadcasts the same packets to all terminals. The right hand side of (43) is minimized by the throughput optimal control policy given by (40)-(42). ${ }^{5}$ From (29) and (33)-(35), the Lyapunov drift (43) satisfies

$$
\Delta_{m}(\mathbf{U}(t)) \leq B-2 \tilde{\epsilon}\left(\sum_{i=1}^{K} U_{i}(t)+U_{R}(t)\right)
$$

for constants $B=m^{2}\left(2+\sum_{i=1}^{K}\left(A_{i}^{\max }\right)^{2}\right)$ and $\tilde{\epsilon}=m \epsilon$, where $\epsilon$ satisfies the flow conditions given by (44)-(46). Then, from (47) the average congestion is bounded as

$$
\limsup _{t \rightarrow \infty} \frac{1}{t} \sum_{\tau=0}^{t-1}\left(\left(\sum_{i=1}^{K} \mathbb{E}\left[U_{i}(\tau)\right]\right)+\mathbb{E}\left[U_{R}(\tau)\right]\right) \leq \frac{B}{2 \tilde{\epsilon}}
$$

and system is strongly stable from congestion bound (48). The throughput optimal control policy (40)-(42) minimizes the right hand side of (43) while satisfying the flow conditions (29) and (33)-(35) such that all rates in the maximum throughput region $\Lambda$ given by (27) can be stabilized.

We can further simplify the throughput optimal control given by (40)-(42). Assume that any superposition of arriving signals is immediately amplified and forwarded by relay $R$ in the next time slot such that relay $R$ transmits, if $\tilde{C} U_{R}(t)>0$ (regardless of the terminal queue backlogs). Provided that $\tilde{C}>0$, this alternative form of throughput optimal control is given by (49)-(51) at time slot $t$ and relies on immediate forwarding by relay $R$ as follows:

${ }^{5}$ If (41) holds with $U_{i}(t)=0$ for any $i=1, \ldots, K$, a dummy packet is transmitted from queue $Q_{i}$ to start the analog network coding session.

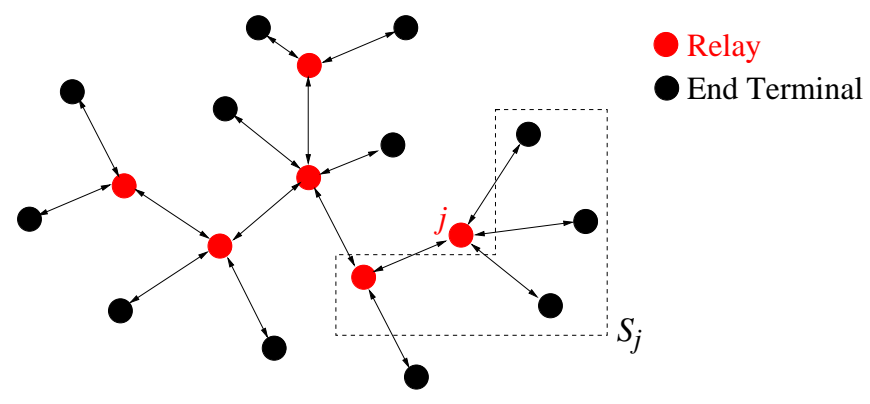

Figure 2: General topology with multiple relays $\mathcal{R}$.

(a) relay $R$ forwards a packet from queue $Q_{R}$, if

$$
U_{R}(t)>0,
$$

(b) terminal $i$ transmits a packet from queue $Q_{i}$, if

$$
U_{R}(t)=0, \quad U_{i}(t) \geq \max \left(\left\{U_{j}(t)\right\}_{j=1}^{K}, \frac{1}{m} \sum_{j=1}^{K} U_{j}(t)\right),
$$

(c) terminals $i=1, \ldots, K$ transmit cooperatively (i.e., they start analog network coding session) over $m$ time slots, if

$$
U_{R}(t)=0, \quad \frac{1}{m} \sum_{j=1}^{K} U_{j}(t) \geq \max \left(\left\{U_{j}(t)\right\}_{j=1}^{K}\right) .
$$

\section{EXTENSION TO MULTIPLE RELAYS}

We extend the single relay case to general multihop operation with multiple relays for broadcast communications, as shown in Figure 2. Define $\mathcal{N}$ as the set of nodes and define $\mathcal{R}$ as the set of relays that have the node degree of more than one (and can perform routing or network coding). Nodes with the node degree of one are called the end terminals.

For relay $j \in \mathcal{R}$, we define $\mathcal{S}_{j}$ as the set of one-hop neighbor nodes of relay $j$ (that have packets to be relayed by relay $j$ ). The node set $\mathcal{S}_{j}$ is predetermined by a set of given paths $\mathcal{P}$ (e.g., by a broadcast tree) such that all nodes are connected with each other through possibly multiple hops. Packets of nodes in $\mathcal{S}_{j}$ must be exchanged over relay $j \in \mathcal{R}$ and there are no cycles allowed in the predetermined set $\mathcal{P}$ of broadcast paths.

For any node $i$, we define $\tilde{\lambda}_{i, j}$ as the total rate of traffic that is carried to the one-hop relay $j \in \mathcal{R}$ (such that $i \in \mathcal{S}_{j}$ ) and further relayed by relay $j$. This total rate consists of the rate $\lambda_{i}$ of self-generated traffic and the rate of relay traffic incoming from neighbor nodes, and it is given by

$$
\begin{aligned}
& \tilde{\lambda}_{i, j}=\lambda_{i}, \quad i \notin \mathcal{R}, i \in \mathcal{S}_{j}, j \in \mathcal{R}, \\
& \tilde{\lambda}_{i, j}=\lambda_{i}+\sum_{l \in \mathcal{S}_{i} \backslash\{j\}} \tilde{\lambda}_{l, i}, \quad i \in \mathcal{R}, i \in \mathcal{S}_{j}, j \in \mathcal{R} .
\end{aligned}
$$

\subsection{Digital Network Coding}

The source and relay traffic can be separated in transmissions. Therefore, we separately define $Q_{i}^{i}$ as the queue at (relay or end terminal) node $i$ for the self-generated packets and $Q_{j}^{i}$ as the queue at relay node $j$ for the relay packets incoming from node $i \in \mathcal{S}_{j}$. The backlogs of queues $Q_{i}^{i}$ and $Q_{j}^{i}$ at time slot $t$ are $U_{i}^{i}(t)$ and $U_{j}^{i}(t)$, respectively. We use 
the notation

$$
\sum_{(m)}\left\{\lambda_{i}\right\}=\sum_{i=1}^{m} \lambda_{i} \quad \text { for } \quad \lambda_{i} \geq \lambda_{j} \quad \text { if } \quad i<j,
$$

which is the sum of the largest $m$ elements in set $\left\{\lambda_{i}\right\}$.

We define $\mathcal{N}_{k}^{j}$ as the set of nodes $\mathcal{S}_{j}, j \in \mathcal{R}$, that a node $k \in \mathcal{S}_{j}$ can overhear, and we define $m_{j}=\left|\mathcal{S}_{j}\right|-1-2 n_{j}$, where $n_{j}$ for $i \in \mathcal{S}_{j}$ is the number of hops a node can overhear on each side from the neighbor nodes of relay $j \in \mathcal{R}$. The flow conditions (10)-(11) for the single relay case can be generalized to multiple relays such that the achievable rates $\lambda_{j} \geq 0, j \in \mathcal{N}$, satisfy

$$
\sum_{\left(m_{j}\right)}\left\{\frac{\tilde{\lambda}_{i, j}}{C_{j}^{i}}\right\}_{i \in \mathcal{S}_{j}}+\frac{\lambda_{j}}{C_{j}}<f_{j}, \quad j \in \mathcal{N}
$$

where the rates $\tilde{\lambda}_{i, j}$ are given by (52)-(53), $f_{j}$ is the total fraction of time allocated to the transmissions of node $j$,

$$
C_{j}=\min _{k \in \mathcal{S}_{j}} C_{j, k}, \quad j \in \mathcal{R},
$$

which is the channel rate for the source packets from relay $j \in \mathcal{R}$ to neighbor nodes $\mathcal{S}_{j}$, or

$$
C_{j}=C_{j, k}, \quad j \notin \mathcal{R}, j \in \mathcal{S}_{k}, k \in \mathcal{R},
$$

which is the channel rate for the source packets from the end terminal $j \notin \mathcal{R}$ to a relay $k \in \mathcal{R}$ such that $j \in \mathcal{S}_{k}$, and

$$
C_{j}^{i}=\min _{k: k \in \mathcal{S}_{j} \backslash\{i\}, i \in N_{k}^{j}} C_{j, k}, \quad j \in \mathcal{R}, i \in \mathcal{S}_{j},
$$

which is the channel rate for the packets of node $i \in \mathcal{S}_{j}$ from relay $j$ to the rest of neighbor nodes $\mathcal{S}_{j} \backslash\{i\}$.

Optimizing the rate rate conditions (55) over $\left\{f_{j}\right\}_{j \in \mathcal{N}}$ yields the maximum throughput region $\Lambda=\left\{\lambda_{j}\right\}_{j \in \mathcal{N}}$. The throughput optimal control policy uses the following differential backlogs (59)-(61) for plain routing by relay nodes and end terminals as well as requires a new formulation of differential backlogs as given by (62) to take into account the network-coded transmissions from relay nodes:

$$
B_{1}^{i, j}(t)=\left[U_{i}^{i}(t)-U_{j}^{i}(t)\right]^{+}
$$

for transmissions from $i$ (where $i \in \mathcal{S}_{j}$ and $i \notin \mathcal{R}$ ) to $j \in \mathcal{R}$,

$$
B_{2}^{i, j}(t)=\left[U_{j}^{i}(t)-\sum_{k: j \in \mathcal{S}_{k} \backslash\{i\}, k \in \mathcal{R} \backslash\{i, j\}} U_{k}^{j}(t)\right]^{+}
$$

for transmissions of packets of $i \in \mathcal{S}_{j}$ from $j \in \mathcal{R}$ to $\mathcal{S}_{j} \backslash\{i\}$,

$$
B_{3}^{j}(t)=\left[U_{j}^{j}(t)-\sum_{k: j \in \mathcal{S}_{k}, k \in \mathcal{R} \backslash\{j\}} U_{k}^{j}(t)\right]^{+}
$$

for transmissions of self-generated packets from $j \in \mathcal{R}$ to $\mathcal{S}_{j}$, and

$$
B_{4}^{i, j}=\frac{1}{m_{j}}\left[U_{j}^{i}(t)-\sum_{k: j \in \mathcal{S}_{k} \backslash\{i\}, k \in \mathcal{R} \backslash\{i, j\}} U_{k}^{j}(t)\right]^{+}
$$

for digital network-coded transmissions from $j \in \mathcal{R}$ to $i \in$
$\mathcal{S}_{j}$. Based on channel rates (56)-(58), we define the weights

$$
\begin{aligned}
& W_{1}^{i, j}(t)=\left\{\begin{array}{cc}
C_{i, j} & \text { if } i \notin \mathcal{R} \text { successfully transmits } \\
\text { to } j \in \mathcal{R}, \text { where } i \in \mathcal{S}_{j}, & \text { otherwise, }
\end{array}\right. \\
& W_{2}^{i, j}(t)=\left\{\begin{array}{cc}
C_{j}^{i} & \text { if } j \in \mathcal{R} \text { successfully transmits } \\
0 & \text { to } \mathcal{S}_{j} \backslash\{i\}, \text { where } i \in \mathcal{S}_{j}, \\
\text { otherwise, }
\end{array}\right.
\end{aligned}
$$

Let $\mathcal{T}(t)$ denote the set of nodes transmitting at time slot $t$. Throughput optimal control to achieve the rate conditions (55) follows from choosing $\mathcal{T}(t)$ to bound the time-average value of total queue length $\sum_{i \in \mathcal{N}} U_{i}^{i}(t)+\sum_{j \in \mathcal{R}} \sum_{i \in \mathcal{S}_{j}} U_{j}^{i}(t)$. This is realized by maximizing the following weighted sum $W(t)$ of differential backlogs based on (59)-(66):

$$
\begin{gathered}
W(t)=\sum_{j \in \mathcal{R}, i \in \mathcal{S}_{j}, i \notin \mathcal{R}} W_{1}^{i, j}(t) B_{1}^{i, j}(t)+\sum_{j \in \mathcal{R}, i \in \mathcal{S}_{j}} W_{2}^{i, j}(t) B_{2}^{i, j}(t) \\
+\sum_{j \in \mathcal{R}} W_{3}^{j}(t) B_{3}^{j}(t)+\sum_{j \in \mathcal{R}, i \in \mathcal{S}_{j}} W_{4}^{i, j}(t) B_{4}^{i, j}(t) .
\end{gathered}
$$

\subsection{Analog Network Coding}

We define $f_{j, 1}$ and $f_{j, 2}$ as the time fractions allocated to transmissions from nodes $\mathcal{S}_{j}$ to relay $j \in \mathcal{R}$, and from relay $j \in \mathcal{R}$ to nodes $\mathcal{S}_{j}$, respectively. The flow conditions (36)(37) for the single relay case can be generalized to multiple relays such that the achievable rates $\lambda_{j} \geq 0, j \in \mathcal{N}$, satisfy

$$
\sum_{\left(m_{j}\right)}\left\{\tilde{\lambda}_{i, j}\right\}_{i \in \mathcal{S}_{j}}<f_{j, 1}, \quad j \in \mathcal{R},
$$

for transmissions of nodes $\mathcal{S}_{j}$ to relay $j \in \mathcal{R}$, and

$$
\sum_{\left(m_{j}\right)}\left\{\frac{\tilde{\lambda}_{i, j}}{\tilde{C}^{j}}\right\}_{i \in \mathcal{S}_{j}}+\frac{\lambda_{j}}{C_{j}}<f_{j, 2}, \quad j \in \mathcal{R},
$$

for (relay and source packet) transmissions of relay $j \in \mathcal{R}$, where $C_{j}, j \in \mathcal{R}$, is given by $(56), \tilde{C}^{j}=\min _{i \in \mathcal{S}_{j}} \tilde{C}_{i}^{j}$, and

$$
\tilde{C}_{i}^{j}=\min _{k: k \in \mathcal{S}_{j} \backslash\{i\}, i \in \mathcal{S}_{j}, i \notin \mathcal{N}_{k}^{j}, j \in \mathcal{R}} C_{i \rightarrow k}^{j} .
$$

In (69), $C_{i \rightarrow k}^{j}, i \in \mathcal{S}_{j}$, is the rate from node $i$ to node $k \in$ $\mathcal{S}_{j}$ over two hops through the amplify-and-forward operation by relay $j$. Optimizing the rate conditions (67)-(68) over $\left\{f_{j, 1}\right\}_{j \in \mathcal{R}}$ and $\left\{f_{j, 2}\right\}_{j \in \mathcal{R}}$ yields the maximum throughput region $\Lambda=\left\{\lambda_{j}\right\}_{j \in \mathcal{N}}$. Relay nodes do not decode packets incoming from the neighboring end terminals and therefore this model corresponds to partial broadcasting only.

Analog network coding requires that relay $j$ keeps all relay packets in a common queue $Q_{j}$. The self-generated packets are separately kept in queue $Q_{i}^{i}$ at any node $i$ such that the transmissions of source and relay packets are separated in time. The backlogs of queues $Q_{j}$ and $Q_{i}^{i}$ at time slot $t$ are $U_{j}(t)$ and $U_{i}^{i}(t)$. The throughput optimal control policy is based on maximizing a weighted sum $W(t)$ of differential 
backlogs that represent the transmissions to relay nodes by plain routing or analog network coding as well as relay transmissions. This extension is similar to digital network coding and it is skipped here for brevity. The maximum stable rates (67)-(68) can be achieved while bounding the time-average value of total queue length $\sum_{i \in \mathcal{N}} U_{i}^{i}(t)+\sum_{j \in \mathcal{R}} U_{j}(t)$.

\section{STABLE THROUGHPUT COMPARISON}

For single relay $R$, consider channel rates $C_{i, R}=1, C_{R, i}=$ 1 and $C_{i \rightarrow j}=1$ for any terminals $i$ and $j$. Network coding is reduced to plain routing, if $\lambda_{i}=0, i=m+1, \ldots, K$, for $\lambda_{1} \geq \ldots \geq \lambda_{K}$. Otherwise, digital and analog network coding improve the sum rate $\sum_{i=1}^{K} \lambda_{i}$ over plain routing by the amount of $\frac{1}{2} \sum_{i=m+1}^{K} \lambda_{i}$ and $\sum_{i=m+1}^{K} \lambda_{i}$, respectively.

The maximum throughput region for $K-1$ terminals includes the maximum throughput region for $K$ terminals except for the case when $\lambda_{K}>0$. Consider common throughput rates $\lambda_{i}=\lambda, i=1, \ldots, K$. Plain routing, digital and analog network coding asymptotically achieve stable rates $\lambda=\frac{1}{2 K}, \lambda=\frac{1}{2 K-1-2 n}$ and $\lambda=\frac{1}{\max (2\lceil K / 2\rceil, 2(K-1-2 n))}$, respectively, if $2 n<K-1$; otherwise $\lambda=\frac{1}{K}$. These stable rates coincide with the throughput rates derived in [12] for the case of saturated queues. The individual rates of different schemes approach each other, as the number of terminals $K$ increases for the finite values of overhearing range $n$.

Next, consider two relay nodes connected in tandem. Each relay is connected to $K$ end terminals. Consider channel rates $C_{i, j}=1$ for any neighbors $i$ and $j$, and $C_{i \rightarrow k}^{j}=1$ for any relay $j$ and two-hop neighbors $i$ and $k$. Each (relay or end terminal) node generates the packet traffic with common rate $\lambda$. Assume that a relay and end terminals connected to its one-hop neighbor relay can overhear each other depending on the hop-distance. However, the end terminals connected to different relays cannot overhear each other. Let $n_{j}=n, j=1,2$. For $2 n<K$, plain routing, digital and analog network coding asymptotically achieve stable rates $\lambda=\frac{1}{5 K+4}, \lambda=\frac{1}{5 K+2-4 n}$ and $\lambda=\frac{1}{4 K+2-4 n}$, respectively.

\section{CONCLUSIONS}

In this paper, we evaluated the maximum throughput region for relay-assisted wireless broadcast under digital and analog network coding. We showed that throughput optimal control results in variations of the maximum differential backlog policy and ensures the stable operation for arrival rates in the maximum throughput region. We started with the case of a single relay and extended the analysis to the simultaneous operation of multiple relays. The results quantify the stable throughput improvement of digital and analog network coding over plain routing. The throughput optimal control schemes rely on centralized scheduling with instantaneous queue backlog information. Future work should look at distributed control with limited or delayed information on queues and channel properties. In addition, the trade-offs of stable throughput with delay and energy measures should be further analyzed in the context of network coding.

\section{REFERENCES}

[1] L. Tassiulas and A. Ephremides, "Stability Properties of Constrained Queueing Systems and Scheduling for Maximum Throughput in Multihop Radio Networks," IEEE Trans. Autom. Control, vol. 37, no. 12, pp. 1936-1949, Dec. 1992.
[2] S. Sarkar and L. Tassiulas, "A Framework for Routing and Congestion Control for Multicast Information Flows," IEEE Trans. Inform. Theory, vol. 48, no. 10, pp. 2690-2708, Oct. 2002.

[3] R. Ahlswede, N. Cai, S. -Y. R. Li, and R. W. Yeung, "Network Information Flow," IEEE Trans. Inform. Theory, vol. 46, no. 4, pp. 1204-1216, July 2000.

[4] S. Katti, S. Gollakota, and D. Katabi, "Embracing Wireless Interference: Analog Network Coding," Proc. ACM SIGCOMM, Kyoto, Japan, Aug. 2007.

[5] T. Ho and H. Viswanathan, "Dynamic Algorithms for Multicast with Intra-session Network Coding," Proc. Allerton Annual Conference on Communication, Control, and Computing, Monticello, IL, Sept. 2005.

[6] Y. E. Sagduyu and A. Ephremides, "Cross-Layer Optimization of MAC and Network Coding in Wireless Queueing Tandem Networks," IEEE Trans. Inform. Theory, vol. 54, no. 2, pp. 554-571, Feb. 2008.

[7] C.-C. Wang and N.B. Shroff, "On Wireless Network Scheduling with Intersession Network Coding," Proc. Conference on Information Sciences and Systems, Princeton, NJ, Mar. 2008.

[8] T. J. Oechtering and H. Boche, "Stability Region of an Efficient Bidirectional Regenerative Half-duplex Relaying Protocol," Proc. IEEE Information Theory Workshop, Chengdu, China, Oct. 2006.

[9] F. Xue, C.-H. Liu, and S. Sandhu, "MAC-Layer and PHY-Layer Network Coding for Two-Way Relaying: Achievable Rate Regions and Opportunistic Scheduling," Proc. Allerton Conference on Communication, Control and Computing, Monticello, IL, Sept. 2007.

[10] Ertugrul N. Ciftcioglu, Aylin Yener, and Randall Berry, "Stability of Bi-Directional Cooperative Relay Networks," Proc. IEEE Information Theory Workshop, Porto, Portugal, May 2008.

[11] A. Eryilmaz and D. S. Lun, "Control for Inter-session Network Coding," Proc. Workshop on Network Coding, Theory and Applications, San Diego, CA, Jan. 2007.

[12] Y. E. Sagduyu, D. Guo, and R. Berry, "On the Delay and Throughput of Digital and Analog Network Coding for Wireless Broadcast," Proc. Conf. on Information Sciences and Systems, Princeton, NJ, Mar. 2008.

[13] Y. E. Sagduyu, D. Guo, and R. Berry, "Throughput Optimal Control for Relay-Assisted Wireless Broadcast with Network Coding," Proc. The First IEEE International Workshop on Wireless Network Coding, San Francisco, CA, June, 2008.

[14] L. Tassiulas and A. Ephremides, "Dynamic Server Allocation to Parallel Queues with Randomly Varying Connectivity," IEEE Trans. Inform. Theory, vol. 39, no. 2, pp. 466-478, Mar. 1993.

[15] F. Xue and S. Sandhu, "PHY-Layer Network Coding for Broadcast Channel with Side Information," Proc. IEEE Information Theory Workshop, Lake Tahoe, CA, Sept. 2007.

[16] D. Bertsekas and R. Gallager, Data Networks, 2nd ed. Prentice Hall, Englewood Cliffs, NJ, 1992.

[17] L. Georgiadis, M. J. Neely, and L. Tassiulas, "Resource Allocation and Cross-Layer Control in Wireless Networks," Foundations and Trends in Networking, vol. 1, no. 1, pp. 1-144, 2006. 